\title{
DISCURSOS, INSTITUCIONES Y SABER EN EL PENSAMIENTO DE MICHEL FOUCAULT
}

\author{
María Cristina Conforti Rojas \\ doi: 10.11144/Javeriana.uph34-69.disf
}

\begin{abstract}
RESUMEN
Este escrito reflexiona acerca del lugar del discurso en el saber y en particular en las instituciones, en el contexto de la enseñanza del filósofo francés Michel Foucault. La pregunta por el discurso conduce a nuestro autor a indagar sobre la relación de este con el saber y el poder. La imbricación de saber y poder pone en marcha una potente y múltiple tecnología de producción que se extiende a toda la sociedad y sus instituciones, pero de manera positiva. Ejemplo de los efectos positivos de la imbricación poder-saber lo encontramos en la institución escolar y en su particular redescubrimiento de las disciplinas. Recogemos, brevemente, humanistas de los siglos XVI y XVII como ejemplos de los efectos positivos de la idea de formación del hombre y de la mujer; así como el ejercitarse en tareas intelectuales en el estudio de las diferentes disciplinas escolares, indispensables para alcanzar el pensamiento crítico, efecto positivo, productivo del aprendizaje escolar.
\end{abstract}

Palabras clave: Michel Foucault; discurso; institución; saber; enseñanza

* Pontificia Universidad Javeriana, Bogotá, Colombia.

Correo electrónico: conforti@javeriana.edu.co

Para citar este artículo: Conforti Rojas, M.C. (2017). Discursos, instituciones y saber en el pensamiento de Michel Foucault. Universitas Philosophica, 34(69), pp. 105-119. ISSN 0120-5323, ISSN en línea 2346-2426. doi:10.11144/Javeriana.uph34-69.disf 


\title{
DISCOURSES, INSTITUTIONS AND KNOWLEDGE IN MICHEL FOUCAULT'S THOUGHT
}

\author{
María Cristina Conforti Rojas
}

\begin{abstract}
This paper reflects on the relation between discourse, knowledge and institutions, in the context of the teachings of the French philosopher Michel Foucault. The question about discourse leads Foucault to inquire about the relation between knowledge and power. The imbrication of knowledge and power that his analysis uncovers sets in motion a powerful and multiple production technology that extends with positive effects to all society and institutions. An example of this is the institution of school and in its particular rediscovery of disciplines. We briefly review the writings of humanists of the sixteenth and seventeenth centuries as examples of the positive effects of the idea of educating men and women in intellectual tasks through the study of school disciplines, indispensable to achieve critical thought, the core of the positive and productive effect of school learning.
\end{abstract}

Key words: Michel Foucault; discourse; institution; knowledge; teaching 
EN ESTE TRABAJO PRETENDO explorar la relación entre los discursos, las instituciones y el saber en Michel Foucault. Elegí estos tres temas porque de ellos se desprende la empresa investigativa filosófica en la que transcurrió la vida de Foucault desde su primer trabajo, titulado Enfermedad mentaly personalidad (1954), hasta las últimas clases, entrevistas y conferencias que impartió hasta su muerte en 1984 .

\section{Subjetividad y discurso}

EMPIEZO POR SUBRAYAR EL escrito El sujeto y el poder ${ }^{1}$ en el que Foucault nos ofrece una síntesis de su trabajo y su legado. En este texto Foucault recoge casi la totalidad de los temas que ha perseguido, sintetizando todas las búsquedas y los propósitos de su investigación a través de una pregunta que vincula dos amplísimos campos de trabajo que lo ocuparon durante años: "Por qué estudiar el poder: la cuestión del sujeto" (Foucault, 2001). Bajo este título considera retrospectivamente su obra y declara:

Me gustaría decir, ante todo, cuál ha sido la meta de mi trabajo durante los últimos veinte años. No he estado analizando el fenómeno del poder, ni elaborando los fundamentos de este tipo de análisis. Mi objetivo, en cambio, ha sido crear una historia de los diferentes modos a través de los cuales los seres humanos se han convertido en sujetos. (p. 241).

La indagación filosófica emprendida por Foucault, que ha pasado por develar el vínculo entre el poder y el saber, lo ha llevado a sostener que nos hemos convertido en sujetos a través de "tres formas de objetivación" (Foucault, 2001, p. 241).

La primera guarda relación con su libro Las palabras y las cosas, en el que estudia "la forma como el hombre aislado o en grupo se haya convertido en objeto de la ciencia, [que] no puede ser considerado ni tratado como un fenómeno de opinión: es un acontecimiento en el orden del saber" (Foucault, 2010, p. 358).

1 El sujeto y el poder, por qué estudiar el poder: La cuestión del sujeto, fue escrito en inglés por Foucault y publicado por primera vez como post-scriptum en el libro de Hubert L. Dreyfus y Paul Rabinow, titulado Michel Foucault Beyond Structuralism and Hermeneutics en 1982 por The University of Chicago Press. 
Dicho acontecimiento toma forma en la particular concepción de la historia de Foucault, en la denominada época clásica, siglos XVII y XVIII. Se pregunta qué hay detrás del sujeto que habla, del sujeto que trabaja y del sujeto que vive, a lo cual responde de forma paradójica y dramática. Su estudio lo lleva a mostrar que del sujeto que habla se desprende la gramática general, la filología y la lingüística; en otras palabras, no el sujeto que habla, sino, antes bien, un saber sobre el hablar, una forma de objetivación. Del sujeto que trabaja se desprende, por su parte, el análisis de la riqueza y de la economía, es decir una forma de "objetivación del sujeto productivo" (Foucault, 2001, p. 241). Por último, la forma de objetivación del sujeto que vive se desprende de los estudios de la historia natural y de la biología. Estas diferentes formas de objetivación producen distintos discursos que pertenecen a diferentes disciplinas y con estos se han pretendido imponer teorías del lenguaje, de la sociedad y de la vida.

La segunda forma de objetivación estudiada por Foucault como un acontecimiento determinante para que hayamos llegado a ser los hombres y mujeres que somos es la que guarda relación con las prácticas divisorias. El sujeto, o bien se divide a sí mismo o es dividido por los otros. Este proceso lo objetiva. Los ejemplos son el loco y el cuerdo; el enfermo y el sano; el criminal y los buenos muchachos (Foucault, 2001, p. 241).

La tercera forma de objetivación es la que guarda relación con la manera "en que el ser humano se convierte a sí mismo, o a sí misma, en sujeto” (Foucault, 2001, p. 242); el ejemplo que ofrece Foucault es el reconocimiento de sí como "sujeto de sexualidad" (Foucault, 2001, p. 242).

En las diferentes formas de objetivación del sujeto, Foucault estudió el vínculo entre poder y saber y las prácticas que se siguieron de estos. A partir de ello creó la historia en las que tuvieron lugar; gracias a estas el filósofo francés concibe la cuestión del discurso. Para él, el discurso es una suerte de medio que pone en movimiento diferentes cosas, pues: "discursos, instituciones, instalaciones arquitectónicas, decisiones reglamentarias, leyes, medidas administrativas, enunciados científicos, proposiciones filosóficas, morales, filantrópicas pertenecen a un conjunto heterogéneo" (Foucault, 1991, p. 128). Foucault descubre que los discursos son un intermedio para la acción, la práctica, en tanto conjunto heterogéneo capaz de producir saber en el orden del discurso, permite ejercer una función productiva y útil en el orden del poder. 
La noción de Foucault acerca del discurso y lo que éste es capaz de producir es presentada de una manera muy original en el desarrollo de su pensamiento y en la forma como lo despliega entre las preguntas por el sujeto que vive, habla y trabaja, tema principal de Las palabras y las cosas, y la objetivación del sujeto en el planteamiento del poder. La bisagra entre la pregunta por el saber y la pregunta por el poder la encontramos en la lección inaugural que pronuncia Foucault el 2 de diciembre de 1970 en el Collège de France, posteriormente publicada con el título El orden del discurso y dedicada al tema del habla, o lo que el discurso es capaz de realizar.

En El orden del discurso Foucault desarrolla la idea de la producción múltiple y temible del discurso que conlleva "poderes y peligros difíciles de imaginar" (Foucault, 1987, p. 11). Con el propósito de desentrañarlos, distingue tres grupos de enrarecimiento del discurso, a saber: el primero, los procedimientos externos de exclusión; el segundo, los procedimientos internos de limitación del discurso, es decir, cuando son los discursos mismos los que ejercen el control, y el tercero, los procedimientos que regulan la distribución de sujetos de enunciación y la apropiación social de los discursos.

En el primer grupo, referido a los procedimientos externos de exclusión del discurso, Foucault sitúa el discurso de lo prohibido, por ejemplo en la esfera de la sexualidad y de la política, en tanto que la vincula con el deseo y el poder. Distingue el discurso de la separación y el rechazo, por ejemplo, entre razón y locura y la oposición entre lo verdadero y lo falso; la verdad del discurso no reside ya en lo que hace, sino, en lo que dice (Foucault, 1987, pp. 11-20).

En cuando al segundo grupo, los "procedimientos internos de limitación del discurso son los discursos mismos los que ejercen el control; procedimientos que juegan un tanto a título de principios de clasificación, de ordenación y de distribución" (Foucault, 1987, p. 21). Entre estos nos encontramos el comentario, la noción de autor y las disciplinas. El comentario -porque en él "lo nuevo no está en lo que se dice, sino en el acontecimiento de su retorno" (Foucault, 1987, p. 24) lo encontramos en los textos religiosos, jurídicos, literarios y, en cierta medida, en los textos científicos. Por su parte, la noción de autor "opera como el principio de agrupación del discurso, como unidad y origen de su significación, como foco de su coherencia". No todos los discursos que circulan van avalados por un autor, por ejemplo, conversaciones cotidianas, decretos, contratos, que tienen necesidad 
de firma pero no de autor. En cuanto al principio de limitación de las disciplinas, éstas se sitúan del lado de la ciencia, se definen el ámbito de estudio, por conjunto de métodos, un corpus de proposiciones, reglas, definiciones, técnicas, todo esto constituye una especie de sistema anónimo, se oponen al comentario y van en búsqueda de la construcción de nuevos enunciados, nuevas proposiciones (Foucault, 1987, pp. 21-32).

El tercer grupo de procedimientos que permite el control de los discursos es el que le impone a los individuos límites y reglas para su enunciación. De acuerdo con Foucault (1987, p. 32), no todo el mundo puede tener acceso al discurso. El ejemplo emblemático del impedimento a los individuos de proferir discursos es el sistema educativo, el sistema de enseñanza en el que el habla es un rito que exige una cualificación de los sujetos, de sus gestos, comportamientos, circunstancias y el conjunto de signos que acompañan el discurso (Foucault, 1987, p. 38). Ejemplo de esta crítica son los discursos religiosos, judiciales, terapéuticos y, en cierta medida, también los discursos políticos.

Foucault denuncia la existencia de una sociedad de discursos, doctrinas y saberes que el sistema de enseñanza se encarga de difundir. En la lección El orden del discurso, Foucault (2010) proporciona la transición de una problemática arqueológica a una genealógica. Esto quiere decir, por ejemplo, que tras la pregunta en las Palabras y las cosas por el hombre que vive, trabaja y habla, Foucault responde con el método arqueológico, es decir, lo sitúa

entre los códigos fundamentales de una cultura -los que rigen su lenguaje, sus esquemas perceptivos, sus cambios, sus técnicas, sus valores, la jerarquía de sus prácticas- en los que se reconocerá en su práctica, y en el otro extremo del pensamiento, las teorías científicas o las interpretaciones de los filósofos explican por qué existe un orden y a qué ley general obedecen (Foucault, 2010, pp. 13-14).

Entre estos dos extremos, los códigos de la cultura y las teorías científicas, existe una región media, una "experiencia desnuda del orden y de sus modos de ser" (Foucault, 2010, p. 14). Explica nuestro autor que se propone estudiar "el lenguaje como era hablado, los seres naturales como eran percibidos, los cambios como eran practicados, es decir, va tras las modalidades del orden" (Foucault, 2010, p. 15).

Foucault sintetiza la transición de la problemática arqueológica a la genealógica o a la segunda forma de objetivación -a la luz del escrito El sujeto y el poder, 
en el que delimita la primera forma de objetivación, a saber, el hombre entendido como objeto de conocimiento y como sujeto cognoscente, signado por "las prácticas divisorias: el loco y el cuerdo; el enfermo y el sano; el criminal y los buenos muchachos" (Foucault, 2001, p. 241)-. El paso de la arqueología a la genealogía tiene lugar en El orden del discurso porque vincula explícitamente el concepto del discurso con el poder y el control, de ahí que el autor plantee que en toda sociedad "la producción de discurso está a la vez controlada, seleccionada y redistribuida por un cierto número de procedimientos que tienen por función conjurar los poderes y peligros" (Foucault, 1987, p. 11).

Ahora bien, la cuestión por el poder y la cuestión del sujeto, el segundo modo de objetivación, lo conducen a preguntarse directamente por la relación entre teoría y práctica, poder y saber; por el modo en que estos se superponen, el modo en que se "implican directamente el uno al otro; que no existe relación de poder sin constitución correlativa de un campo de saber, ni de saber que no suponga y no constituya al mismo tiempo relaciones de poder" (Foucault, 2009, p. 37). Esta interrelación, que Foucault desarrolla en su libro Vigilar y castigar, inaugura un amplísimo y fecundo campo de investigación. Declara que el objetivo de dicho libro es "hacer una historia correlativa del alma moderna y de un nuevo poder de juzgar; una genealogía del actual complejo científico-judicial" (Foucault, 2009, p. 32), es decir, busca hacer una historia de los modos de objetivación y producción de subjetividad que la relación entre poder y saber pone en marcha y de un nuevo poder de juzgar que redescubre las disciplinas, gracias a las cuales tienen lugar las instituciones.

La disciplina es una tecnología, una anatomía política del detalle. [...] La era clásica no la ha inaugurado; el detalle era desde ya hacía mucho tiempo una categoría de la teología y del ascetismo. [...] En esta gran tradición de la eminencia del detalle vendrán a alojarse, sin dificultad, todas las meticulosidades de la educación cristiana, de la pedagogía escolar o militar y, finalmente, de todas las formas de encauzamiento de la conducta (Foucault, 2009, pp. 161-162).

Foucault ve que el potenciamiento de esta tecnología en el ejercicio del poder se amplía del ascetismo cristiano a un contexto más general de la sociedad, que toca la política, la pedagogía, la medicina, al ámbito "de la tecnología del poder sobre el cuerpo, que la tecnología del alma -la de los educadores, de los 
psicólogos y de los psiquiatras- [...] se entiende por ello hacer la historia del pasado en términos del presente" (Foucault, 2009, p. 40).

La nueva tecnología, al articular poder y saber a través de la tecnología disciplinaria, hace que

nazca un arte del cuerpo humano que no tiende únicamente al aumento de sus habilidades, ni tampoco a hacer más pesada su sujeción, sino a la formación de un vínculo que, en el mismo mecanismo lo hace tanto más obediente cuanto más útil (Foucault, 2009, p. 160).

El sujeto-objeto de esta tecnología disciplinaria es el cuerpo dócil, el que "puede ser sometido, que puede ser utilizado, que puede ser transformado y perfeccionado" (Foucault, 2009, p. 159). Se trata de un ejercicio del poder que va en pos de la perfección que se encuentra en el detalle, en la clausura, en el ocupar cada uno su lugar, en la ocupación del tiempo, ya sea en el taller, en la escuela, en la formación militar, etc., por la "organización de una nueva economía del tiempo de aprendizaje” (Foucault, 2009, p. 170).

Esta última organización da lugar a la institución escolar. A la escuela que conocemos, de la que nos reconocemos como herederos, la que ha funcionado bajo la forma de una tecnología disciplinaria, pero también ha organizado y transmitido las ciencias. No podemos evitar incomodarnos ante la descripción de las tecnologías disciplinarias; no obstante, podemos oponer a la incomodidad que nos produce la idea de que somos producto de una tecnología disciplinaria el propósito general que enuncia Foucault en su libro Vigilar y castigar, a saber, mostrar que los efectos de todos estos mecanismos no han sido negativos, como si su propósito fuera "reprimir, impedir, excluir, suprimir, sino que están ligados a toda una serie de efectos positivos y útiles" (Foucault, 2009, p. 34).

\section{La escuela una institución que va más allá de las disciplinas}

LA ESCUELA ES UNA DE LAS InStituciones de la sociedad de las que podemos derivar efectos positivos y útiles. A continuación, presentaré una breve distinción entre la escuela como institución y la abundante literatura escrita sobre el tema. Sobre esta última cabe mencionar: los tratados de educación del siglo XVI, en el que encontramos autores como Montaigne (1533-1592) y Juan Luis Vives (1493-1540), los tratados de pedagogía de los jesuitas y Lutero (1483-1546), 
que indudablemente pensaron la educación y en sus fines de una manera aguda, buscando responder a las necesidades de su tiempo. Estos autores llamaron disciplinas a las asignaturas que había que aprender, haciendo énfasis en el estudio de las letras, del latín y de las matemáticas; así mismo, como humanistas consideraron que el dominio de la lengua guardaba una cierta relación con la intimidad de cada uno, por lo que su cultivo sería una potente herramienta para nuestro propio gobierno. En esta literatura predominó también una temática recurrente dirigida a la educación del príncipe, que, ejercía el poder, como afirma Foucault (2016), "sobre el gobierno de las almas y las conductas, el problema de la pastoral católica y protestante” (p. 10), así como numerosa literatura que abundaba en consejos para ser buen gobernante, para ser buen cristiano, consideraban la necesidad de que el príncipe se educara y escuchara los consejos con el fin de que entendiera la diferencia entre el reino del gobierno civil y el reino del gobierno espiritual. Otro elemento común en esta literatura es la defensa de una educación que debía impartirse a los hombres que se consideraban destinados a gobernar, ya fuera en el ámbito civil o en el espiritual.

Heredera de este espíritu, y con una importancia considerable en lo que respecta a la historia de la escuela, es la obra pedagógica de Jan Amós Comenius, quien nació en Moravia Checa en el año 1592 y murió en Ámsterdam en 1670. Su obra Didáctica Magna fue publicada en checo en 1632 y en latín en 1640. En este libro, Comenius (2012, p. 55) plantea que el hombre debe ser formado para la humanidad. Cabe resaltar la idea que presenta acerca de la formación del hombre en cuanto hombre, pues Comenius considera el mismo principio moral y religioso de los pedagogos del siglo XVI, a saber, que el fin del hombre está en otra vida, en la vida eterna; sin embargo, se distancia de estos en torno a elementos fundamentales de la educación tales como: que esta debe empezar en la primera edad (Comenius, 2012, p. 61), que la juventud debe educarse reunida por el ejemplo que deben tomar unos de otros (Comenius, 2012, p. 69), y que se debe contar con la coeducación. En esta vía, defiende que no hay razones para excluir a las niñas de la escuela (Comenius, 2012, pp. 70-74) y que la enseñanza en la escuela debe ser universal, es decir, debe ser enseñado todo a todos (Comenius, 2012, p. 75). La escuela debe ser según Comenius "un verdadero taller de hombres" (Comenius, 2012, p. 82), su fin: 
preparar para adquirir un conocimiento verdadero y sólido, no falso y superficial; es decir, que el animal racional, el hombre, se guíe por su propia razón no por la ajena; no se limite únicamente a leer y aprender en los libros pareceres y consideraciones ajenos de las cosas o a retenerlos en la memoria y recitarlas, sino que sea capaz de penetrar hasta la médula de las cosas y conocer de ellas su verdadera significación y empleo. En igual medida hay que atender a la solidez de costumbres y piedad (Comenius, 2012, p. 89).

Comenius ve en la escuela fines positivos y útiles, ya que el trabajo que se da en esta tiene como objetivo la formación del hombre en cuanto hombre, entendido este como un sujeto capaz de conocimiento de las cosas y de pensar por sí mismo. Comenius en su didáctica ofrece muchos ejemplos y argumentos por los cuales busca persuadir a sus coetáneos de la importancia de la escuela para la educación de los niños, particularmente de las niñas, que, en el siglo XVII, encontraban, aún, mucha resistencia. El prejuicio dominante para la educación de la mujer consistía en que debía ser instruida únicamente en asuntos morales, por ejemplo para Vives "el cuidado exclusivo de la mujer es la pudicia" (García Hoz, 1994, p. 141), es decir, a la mujer, solo había que enseñarle a guardar y observar la honestidad en acciones y palabras. Por el contrario, Comenius (2012) sostenía que "no existe ninguna razón por la que el sexo femenino deba ser excluido en absoluto de los estudios científicos ya se den en lengua latina, ya en idioma patrio" (p. 73). Además, pensaba que la única manera de prevenirnos del error y del vicio, tanto a hombres como a mujeres, era la asistencia y los ejercicios de educación que se practicaban en la escuela. Esto último lo ilustra de la siguiente manera:

para el hombre fue el paraíso una escuela manifiesta antes de la caída, y poco a poco aprovechaba de ella. Pues, aunque al primer hombre, en cuanto fue producido, no le faltó ni la marcha ni el lenguaje ni el raciocinio, sin embargo, carecía del conocimiento de las cosas que proviene de la experiencia, como lo atestigua el coloquio de Eva con la serpiente, en el que, si ella hubiera tenido mayor experiencia, no habría accedido tan sencillamente sabiendo que no era propio el lenguaje de tal criatura y, por lo tanto, que existía engaño (Comenius, 2012, p. 57).

Comenius considera indispensables para la educación, tanto de hombres como de mujeres, la experiencia, el ejercicio del raciocinio y del lenguaje y el conocimiento de las cosas para no dejarnos arrastrar por el error, ya que son 
herramientas fundamentales para prevenirnos de los errores y para la formación de la moral. Concibe desde el siglo XVII la importancia de que las niñas se eduquen junto con los niños, con ello, presenta una nueva dimensión de la institución escolar, la cual se centra en la enseñanza y el aprendizaje, pues, para él, el fin de la educación es enriquecer la vida, potenciar las capacidades, formar el carácter y la personalidad de un individuo, que la educación recibida permee la conducta. Concibió una institución para la formación del hombre que exigía también condiciones y métodos de enseñanza.

El fin de la escuela, para Comenius, es potenciar la inteligencia, la imaginación, la memoria, la capacidad de aprender y de pensar, así como educar en las buenas costumbres. El fin de cualquier institución educativa sería fortalecer y profundizar la formación del hombre en cuanto hombre.

La idea de la educación del hombre en tanto hombre ha dado lugar a dos dimensiones que se complementan y en cierto sentido se oponen, de un lado, las concepciones espirituales y de otro, las disciplinas. La institución escolar se ha encargado de moldear cabezas, corazones y conductas. Lo ha hecho a través de la organización y transmisión del saber, de métodos de enseñanza, así como de reglamentos para modular conductas y modos de ser. Es difícil no reconocerse uno mismo como heredero de las enseñanzas y de la tecnología disciplinaria que ha ejercido la escuela, entendida esta como una institución con la obligación de ser productiva para el cuerpo social. El ejercicio de la tecnología disciplinaria regular, constante, rigurosa con sus respectivos mecanismos de premios, promoción y sanción han convertido a la escuela en una muy productiva institución de la sociedad. Con el propósito de afrontar la incomodidad que nos produce el reconocernos como un producto de la tecnología disciplinaria, el presente artículo propone considerar la salida que presenta Foucault: oponer resistencia, para él significa la "lucha perpetua y multiforme, más que la dominación lúgubre y estable de un aparato uniformador" (Foucault, 2001, p. 407). "Lucha perpetua y multiforme frente a un aparato uniformador", creemos que significa hacer uso de la inteligencia que se ha cultivado en los numerosos ejercicios escolares, en las lecturas, en la forma en que se ha aprendido a actualizar las capacidades, por ejemplo, en el discernimiento sobre lo que nos conviene, lo que debemos hacer, en lo que creemos, pues pensar involucra desplegar destrezas, habilidades, disposiciones, clasificaciones y observaciones. Todas estas competencias se ejercitan en la escuela y 
suponen un entrenamiento, un aprender. Dicho ejercicio de las capacidades intelectuales, del pensar, debe llevarnos a establecer relaciones, a profundizar y ampliar la ciencia, pero sobre todo a asumirnos como sujetos. Oponer resistencia significa, entonces, que el hecho de habernos educado, nos impone la obligación de pensar, que el pensar debe conducirnos a considerar nuestra dimensión social y al hecho de que somos objetos de educación pero también sujetos sociales. Esta es, creo, la enseñanza que aprendimos de Kant en sus escritos sobre la Ilustración, en particular, en su “Respuesta a la pregunta: ¿qué es la Ilustración?”. En este trabajo Kant nos dice que la ilustración significa "salir de la culpable minoría de edad” (Kant, 1999, p. 63). Después, el filósofo alemán aclara qué significa la salida de esa culpable incapacidad y delimita el significado de la ilustración.

Ilustración y autonomía, inteligencia y carácter son condiciones necesarias para ejercer el pensamiento crítico sin sujeción a autoridad alguna. No obstante, Kant muestra de diferentes maneras que nuestras acciones tienen implicaciones sociales y políticas de hondo calado. Por un lado, Kant nos urge a no aceptar tutela de nadie y, por otro, nos insta a obedecer en tanto que somos miembros de una sociedad y tenemos obligaciones con esta; en este punto se encuentra la disyuntiva enunciada por Foucault, la cual hace referencia a la íntima relación entre la ilustración y las disciplinas. En Vigilar y castigar afirma que "las luces, que han descubierto las libertades, inventaron también las disciplinas” (Foucault, 2009, p. 255).

La respuesta de Kant a la pregunta por la Ilustración, cuyo significado es "la de hacer uso público en todas partes de la razón”, propone tres ejemplos a propósito de lo que es razonar y obedecer: "El oficial dice: ¡No razones, sino ejercítate! El consejero de hacienda: ¡No razones, sino paga! El clérigo: ¡No razones, sino cree!" (Kant, 1999, p. 65). Ejercitarse, pagar y creer tienen que ver con el puesto civil o la función que se deba desempeñar, con el pertenecer a una sociedad concreta en la que se cumple una función laboral particular y se tienen obligaciones con arreglo a las normas; es en este sentido en el que quiero resaltar, del pensamiento de Kant, que nuestras acciones tienen implicaciones en la sociedad que demandan nuestro asentimiento, nuestra obediencia. El filósofo alemán formula esta idea de la siguiente manera: “irazona tanto como quieras y sobre lo que quieras; solo que obedece!” (Kant, 1999, p. 65).

Foucault (2006, p. 54) reanuda la discusión en torno a la Ilustración y hace énfasis en la problematización de nuestro presente, lee la Ilustración en clave de 
actualidad; de este modo renueva la pregunta por la Ilustración kantiana y la reconduce a su investigación personal, a la cuestión del sujeto. Así, propone cuestiones como: ¿de qué manera conducirse? ¿qué hacer? ¿qué pensar? o ¿cómo nos convertimos a nosotros mismos en sujetos autónomos? ¿quiénes somos? Siguiendo a Gros (2007), dichas preguntas las plantea en modo "singular e histórico ¿quiénes somos? Significa, a la vez, ¿de qué síntesis históricas está constituida nuestra identidad? y ¿cómo podríamos ser de otro modo?” (p. 127).

Foucault (2006) reconduce su respuesta al ámbito de la libertad, y al igual que Kant al de la moral, a la "reactivación de una actitud; que puede caracterizarse como crítica” (p. 86). Para Foucault el verdadero significado de la Ilustración tiene que ver con que "intentemos el análisis de nosotros mismos en tanto que seres históricamente determinados por la Ilustración” (Foucault, 2006, p. 87). Esta actitud, este éthos, debe conducirnos según nuestro autor a investigaciones detalladas, por ejemplo:

cuando alguien comete algo así como lo que llamaríamos un bello crimen, nadie piensa que ello pueda ser el resultado de una especie de genio sino que es obra de la locura. La relación entre la locura y el crimen, la belleza y el arte es muy enigmática. Nuestra tarea, es intentar comprender por qué consideramos que esta relación es incuestionable (Foucault, 1999, pág. 70).

El anterior ejemplo, tomado de una entrevista realizada a Foucault, enseña en qué consiste para él hacer investigaciones precisas. Ir al caso y a la singularidad no solamente nos pone en la senda de la ilustración, sino que marca una ruptura con los humanismos, pues estos tienen una concepción universal del hombre, distinta de los nuevos descubrimientos de las luces, las libertades y las disciplinas.

El análisis de nosotros mismos no podemos hacerlo sin una crítica permanente, exige elementos de transformación social, unos tipos de instituciones políticas, unas formas de saber, unos proyectos de racionalización de los conocimientos y de las prácticas, que en todo caso conducen a una forma de reflexión filosófica que concierne fundamentalmente a una relación reflexiva con el presente (Foucault, 2006, p. 87).

Quiero terminar resaltando el hecho fundamental de la Ilustración que, me parece, coincide con la tarea de la filosofía y la educación: el intento, el aprendizaje y el ejercitar el pensamiento así como el perseguir nuestra propia autonomía en el reconocimiento de que somos sujetos sociales. 
Foucault, M. (1987). El orden del discurso. (Trad. A. González). Barcelona: Tusquets Editores.

Foucault, M. (1991). El juego de Michel Foucault. En Saber y verdad (pp. 127162). (Trad. Á. Gabilondo). Barcelona: Paidós.

Foucault, M. (1999). Verdad y poder. En M. Foucault, Estrategias de poder. Obras Esenciales Vol. II (pp. 41-55). (Trads. Á. U. Fernando \& J. Varela). Barcelona: Paidós.

Foucault, M. (2001). El sujeto y el poder. En H. L. Dreyfus, \& P. Rabinow (Eds.). Michel Foucault: más allá del estructuraismo y la hermenéutica (pp. 241-259). (Trad. R. Paredes). Buenos Aires: Ediciones Nueva Visión.

Foucault, M. (2001). Pouvoir et savoir. En M. Foucault, D. Defert, F. Ewald, \& J. Lagrange (Eds.), Dits et écrits (1976-1988) Vol. II (pp. 399-414). París: Quarto Gallimard.

Foucault, M. (2006). ¿Qué es la Ilustración? En Sobre la Ilustración (pp. 71-97). $2^{a}$ edición (Trads. J. D. Higuera, E. Bello, \& A. Campillo). Madrid: Tecnos.

Foucault, M. (2006). Seminario sobre el texto de Kant "Was ist Aufklärung?". En Sobre la ilustración (pp. 53-69). 2a edición (Trads. J. D. Higuera, E. Bello, \& A. Campillo). Madrid: Tecnos.

Foucault, M. (2006). Seguridad, territorio y población. Curso en el Collège de France (1977-1978). (Trads. M. E. Snellart \& H. Pons). Buenos Aires: Fondo de Cultura Económica.

Foucault, M. (2009). Vigilar y castigar: nacimiento de la prisión. $2^{\mathrm{a}}$ edición revisada (Trad. A. Garzón del Camino). México: Siglo XXI.

Foucault, M. (2010). Las palabras y las cosas: una arqueología de las ciencias humanas. $2^{a}$ edición (Trad. E. Frost). México: Siglo XXI.

Comenius, J. A. (2012). Didáctica Magna. (S. López Peces, Trad.) Madrid.

Akal.Kant, I. (1999/1784). Respuesta a la pregunta: ¿qué es la Ilustración? En En defensa de la Ilustración (pp. 63-71). (Trads. J. Alcoriza \& A. Lastra). Barcelona: Alba Editorial. 
Gros, F. (2007). Michel Foucault. (Trad. I. Agoff). Buenos Aires: Amorrortu Editores S. A.

García Hoz, V. (1994). Juan Luis Vives, pedagogo de Occidente. En J. Château

(Ed.), Los grandes pedagogos (pp. 34-52). México: Fondo de Cultura Económica. 